\title{
Simulating Ellerman bomb-like events
}

\author{
S. Danilovic \\ Max-Planck-Institut für Sonnensystemforschung, Justus-von-Liebig-Weg 3, 37077 Göttingen, Germany \\ e-mail: danilovic@mps.mpg.de \\ Received 6 January 2017 / Accepted 29 March 2017

\begin{abstract}
Context. Ellerman bombs (EB) seem to be a part of a whole spectrum of phenomena that might have the same underlying physical mechanism: magnetic reconnection.

Aims. The aim of this study is to investigate whether the proposed mechanism, applied to the circumstances of EBs, produces the observed characteristics.

Methods. To this end, comprehensive three-dimensional magnetohydrodynamic (3D MHD) simulations were used. Two different cases are presented: the quiet Sun and an active region.

Results. Both runs confirm that EB-like brightenings coincide with hot and dense plasma, which is in agreement with predictions of $1 \mathrm{D}$ and 2D modellings. The simulated EB-like phenomena assume the observed flame-like form which depends on the complexity of the ongoing reconnection and the viewing angle. At the layers sampled by $\mathrm{H} \alpha$-wings, near temperature minimum and below, the magnetic field topology seems always to be the same. The field lines there trace the base of the current sheet and the reconnected $\Omega$-loops.

Conclusions. The EB features are caused by reconnection of strong-field patches of opposite polarity in the regions where the surface flows are the strongest. The weakest cases among them can be reproduced quantitatively by the current simulations.
\end{abstract}

Key words. Sun: magnetic fields - Sun: photosphere

\section{Introduction}

Ellerman bombs (EBs; Ellerman 1917) are defined as transient brightenings visible in the extended wings of the $\mathrm{H} \alpha$ line. $\mathrm{Re}-$ cently, these features have attracted a lot of attention for two main reasons.

The first of these reasons is the considerable challenge they pose to models because of their specific characteristics. Highresolution observations show that they appear in the shape of a flame that seems to be rooted in the intergranular lanes (Hashimoto et al. 2010; Watanabe et al. 2011). This suggests that their formation begins very low, near the surface. However, 1D and 2D modelling strongly suggests that only a temperature increase starting at heights of a few hundred $\mathrm{km}$ above the solar surface can produce the observed $\mathrm{H} \alpha$ line profile without continuum brightening (Kitai 1983; Fang et al. 2006; Socas-Navarro et al. 2006; Bello González et al. 2013; Berlicki \& Heinzel 2014; Hong et al. 2014; Grubecka et al. 2016). Ellerman bombs also have signatures in $\mathrm{Ca}$ II $\mathrm{H}$ and Ca II IR $854 \mathrm{~nm}$ lines (Matsumoto et al. 2008; Vissers et al. 2013; Rezaei \& Beck 2015), but not in Na I D1 and Mg I b2 lines (Rutten et al. 2015). This indicates that temperatures are high enough for neutral metals to be ionized, but not so high that they loose their visiblity in chromospheric lines. In some cases, however, EBs can also be traced in observables which should sample temperatures orders of magnitude higher (Vissers et al. 2015; Tian et al. 2016; Libbrecht et al. 2017).

The second reason is that they seem to be a part of a whole spectrum of phenomena that might have the same underlying physical mechanism. On the one hand, on the low-energy end of that spectrum are quiet-Sun Ellerman-like brightenings (QSEBs; Rouppe van der Voort et al. 2016). As EBs, these features also produce emission in $\mathrm{H} \alpha$ wings, but they are less bright and smaller in size. On the other hand, at the high-energy end of the spectrum lie the IRIS bombs (IBs; Peter et al. 2014) which in some cases coincide with EBs (Tian et al. 2016), but seem to point to much higher temperatures. The specific spectral signatures indicate local heating in the photosphere or lower chromosphere to $\approx 8 \times 10^{4} \mathrm{~K}$.

Observations show that EBs are preferably formed in young emerging active regions (Bruzek 1968; Rutten et al. 2013; Schmieder et al. 2014). They usually, but not exclusively, appear in series where serpentine field lines emerge in what are known as bald patches (BPs), i.e. dips in magnetic field lines (Pariat et al. 2004, 2006, 2009, 2012). This scenario was supported by the ideal MHD simulations (Isobe et al. 2007; Archontis \& Hood 2009), which generated the temperature and density increase of the order of magnitude that was needed to reproduce the observed $\mathrm{H} \alpha$ wings, but did not allow any quantitative comparison with observations, due to their simplified treatment of the plasma physics.

In Danilovic et al. (2017), we quantitatively compared our 3D comprehensive MHD simulations with high-resolution observations and showed, for the first time, that serpentine-like emergence indeed produces EB-like phenomena. In that paper we focused on the dynamics of simulated EB-like events and their signatures in photospheric neutral iron lines. The current paper further explains what causes their flame-like morphology and shows the similarities between the quiet Sun and active region cases. 

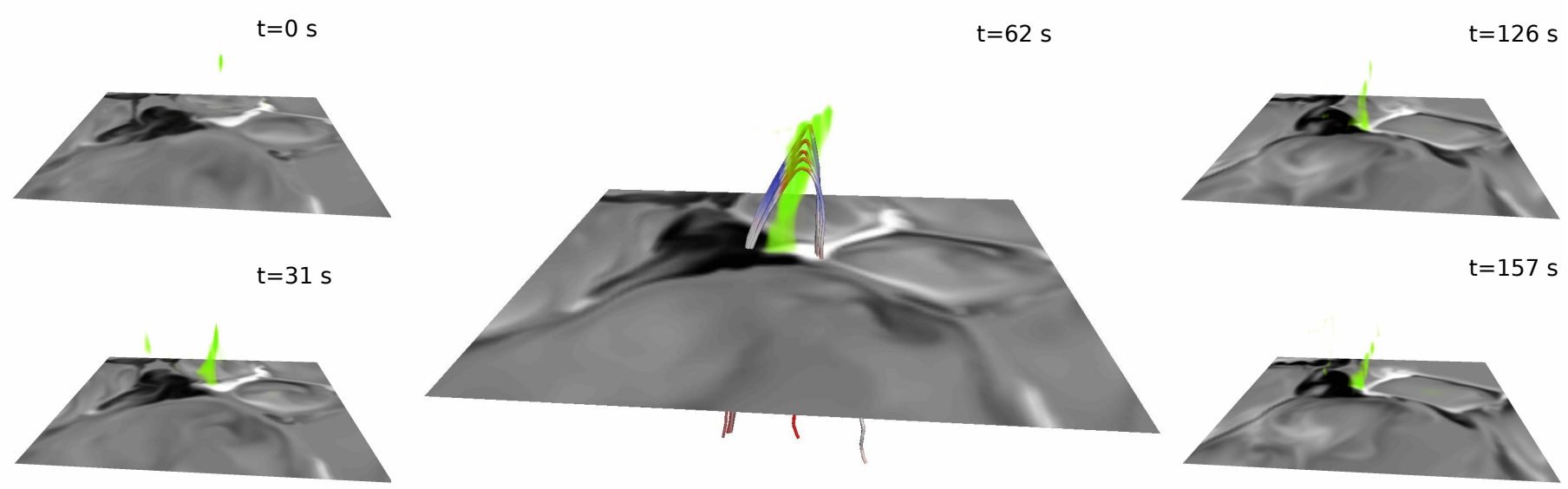

Fig. 1. Results from the weak-field MURaM simulation at half-minute intervals, ordered along columns. The grey scale in the horizontal planes shows the vertical component of the magnetic field at the geometrical height where $\langle\tau\rangle=0.1$. The third sample is enlarged for better visibility. Green denotes volume rendering of temperature in the range $T=5700-6500 \mathrm{~K}$ and outlines the site with most ohmic heating. Some magnetic field lines are shown in the middle panel, colour-coded according to vertical gas velocity (downflows red, upflows blue). The sequence illustrates that the cancellation of opposite-polarity field concentrations produces heated features with EB-like behaviour.

\section{MURaM simulations}

Two essentially different numerical experiments are performed with the 3D MHD MURaM code (Vögler et al. 2005; Rempel et al. 2009) to simulate the conditions of the quietSun and active regions. The runs are variants of those used in Danilovic et al. (2015). Ambipolar diffusion and Hall currents are both taken into account in the induction equation because they might be important in the photosphere for dynamics on very small scales, especially in regions with large magnetic field gradients (Cheung \& Cameron 2012). Non-grey radiative transfer is also included (Vögler et al. 2004; Beeck et al. 2012).

\subsection{Quiet Sun case}

The first run presents a case rather similar to the one presented in Nelson et al. (2013). The computational domain measured $6 \times 6 \times 1.68 \mathrm{Mm}$ at about $700 \mathrm{~km}$ above the $\tau_{5000}=1$ surface (continuum optical depth at $\lambda=5000 \AA$ ) and with a spatial resolution of 10 and $14 \mathrm{~km}$ in the horizontal and vertical directions, respectively. The initial magnetic field at the simulation start was a $2 \times 2$ checkerboard pattern of bipolar vertical magnetic field with height-independent strength of $B=200 \mathrm{G}$.

When the simulation had evolved over approximately $15 \mathrm{~min}$ of solar time, a horizontal flux sheet was inserted at a depth of $300 \mathrm{~km}$ below mean optical depth unity. The field strength profile in the vertical direction across the sheet was defined as a Gaussian with a FWHM of $110 \mathrm{~km}$ and a maximum value $1 \mathrm{kG}$. We then let the simulation run for an additional 30 min of solar time, and saved snapshots at half-minute intervals.

The collected snapshot were used to synthesize emergent profiles of $\mathrm{H}_{\alpha}$ and other lines along every column. In order to facilitate direct comparison with the $\mathrm{H}_{\alpha}$ observations of Vissers et al. (2013) the same spectral sampling and smearing of $66 \mathrm{m \AA}$ was used as in the SST observations. Synthetic images in the line wing were produced with the SPINOR code (Frutiger 2000; Frutiger et al. 2000), and a more detailed profile synthesis was performed along columns with the RH code of Uitenbroek (2001). Both codes produced qualitatively similar results, but minor differences arose from different representations of the hydrogen atom and/or different numerical schemes.
In SPINOR, linear Stark broadening was added as prescribed by Rutten (2016). Because of this, the focus in this paper is given only to SPINOR output.

Figure 1 shows the event that produced the strongest brightening in the $\mathrm{H}_{\alpha}$ wing in this simulation run. It corresponds to a sizeable temperature increase that occurs at the site of the cancellation of two magnetic features of opposite polarities. Figure 1 outlines this feature by colour-coding the temperature increase in the region where the largest ohmic heating occurs. Figure 2 displays the same feature as it appears in synthetic $\mathrm{H}_{\alpha}$ wing images, synthetic $\mathrm{H}_{\alpha}$ Dopplergrams, and Fe I $6301 \AA$ magnetograms, viewed from the side along the slanted $\mu=0.66$ line of sight in the four azimuthal quadrants. This means that the filtergrams are synthesized in the way that the heliocentric angle is perserved, but the direction of the "limb" is changed. The limb direction is up, right, left, and down (clockwise) in the four subpanels shown for each intance during the feature's evolution. The feature appears as an upright "flame" rooted at the surface location where the cancellation occurs, very similar to the morphology described in Watanabe et al. (2011) and Vissers et al. (2013).

The synthetic $\mathrm{H}_{\alpha}$ Dopplergrams in the second column of Fig. 2 suggest downflow at the base and upflow higher, at all azimuthal perspectives. The magnetograms in the third column of Fig. 2 show that the small patch of positive polarity that is present at the base of the green feature in Fig. 1 is not clearly visible in the slanted view. The region around the EB-like feature actually appears unipolar when observed from most angles. This hiding implies that even when no bipolarity is observed, there may actually be enough that cancellation produces EB-like phenomena.

Figure 3 shows how this EB-like feature would look at disk centre with the spatial resolution of the simulation (top row) and at the SST resolution (middle row). Each panel is grey-scaled independently. At the SST resolution the feature's brightness contrast is reduced considerably. At disk centre it would not be very distinct from the brightest network field concentrations, but in limbward viewing its flame-like appearance would still stand out. In the bottom panels the magnetograms are at the same resolution as in the simulation; they illustrate how complex the small scale of the field topography is which causes this feature. 

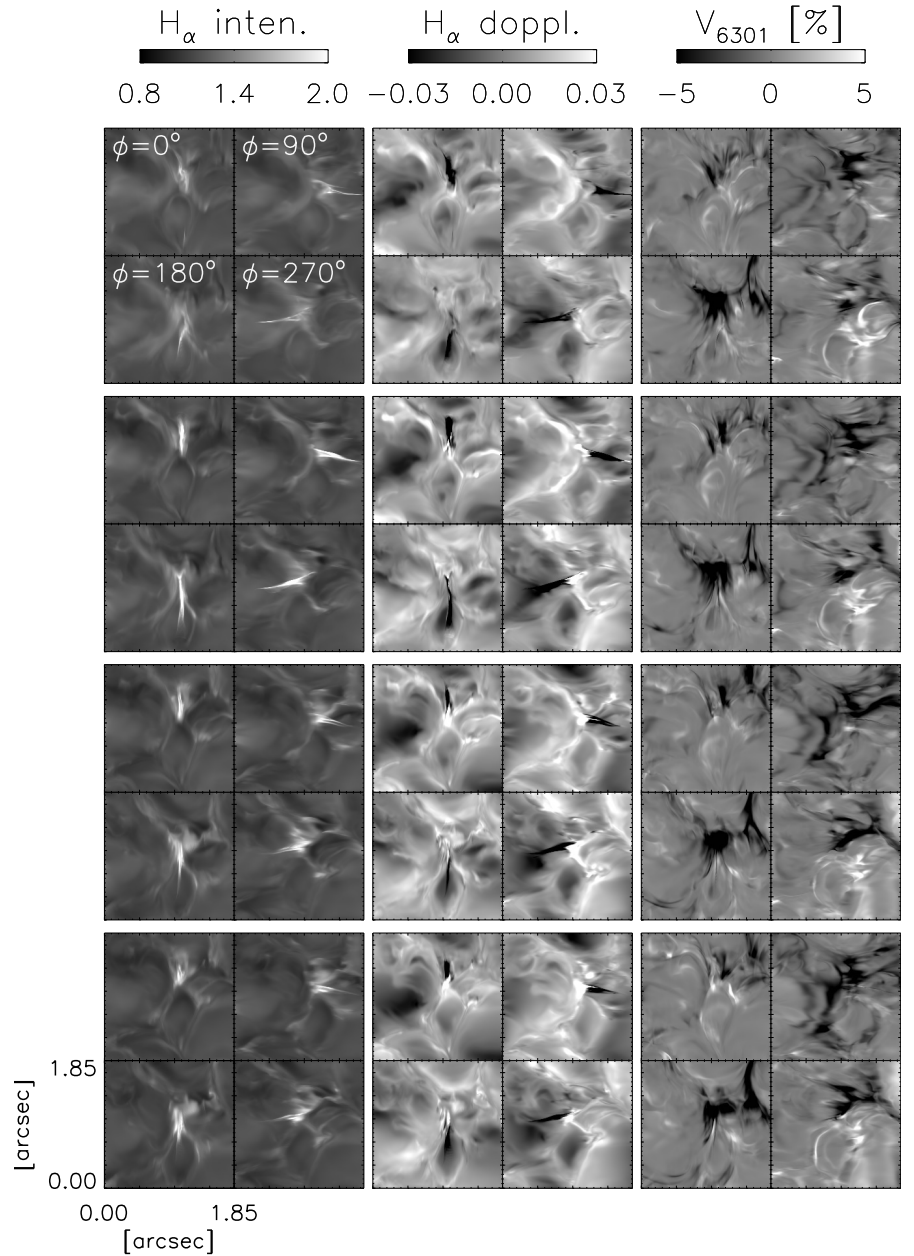

Fig. 2. Details of the EB-like feature for the last four samples in Fig. 1, ordered top to bottom. For each sample time, the first column shows a quartet of synthetic intensity images in the $\mathrm{H}_{\alpha}$ wing at $\Delta \lambda=-0.11 \mathrm{~nm}$ from line centre at viewing angle $\theta=49^{\circ}(\mu=0.66)$, from four different azimuthal viewing directions $\phi$ as specified. The intensity is normalized to the mean continuum intensity over the whole field of view. The second column shows corresponding synthetic $\mathrm{H}_{\alpha}$ Dopplergrams obtained by subtracting normalized blue-wing intensity from red-wing intensity at $\Delta \lambda= \pm 0.11 \mathrm{~nm}$. The third column shows corresponding synthetic magnetograms obtained from the Fe I $6301 \AA$ line at $\Delta \lambda=-48 \mathrm{~m} \AA$ from line centre. The values indicate the amount of normalized circular polarization.

Since a numerical simulation, as distinct from observations, also permits us to diagnose what actually happens below the surface, the vertical cuts through the EB-like feature in the $t=62 \mathrm{~s}$ snapshot are shown in Fig. 4. Their location is specified in the left top and bottom panels of Fig. 3. The third panel shows how this cut samples the two opposite-polarity magnetic concentrations, whose partial cancellation caused the EB-like feature. Higher up, these magnetic features happen to lie out of the selected cut plane, so that especially the orange feature in the third panel of Fig. 4 shows an abrupt stop, due to end of sampling.

The first panel of Fig. 4 shows a substantial temperature increase in and especially just above the sampled part of the righthand magnetic concentration, up to $T=8500 \mathrm{~K}$ at $h \approx 300 \mathrm{~km}$, but already starting at the surface (with $h=0$ defined by $\left.\tau_{5000}=1\right)$.

The second panel of Fig. 4 shows the presence of the two magnetic concentrations as relatively tenuous "fluxtubes". However, a local density increase is present above the second one
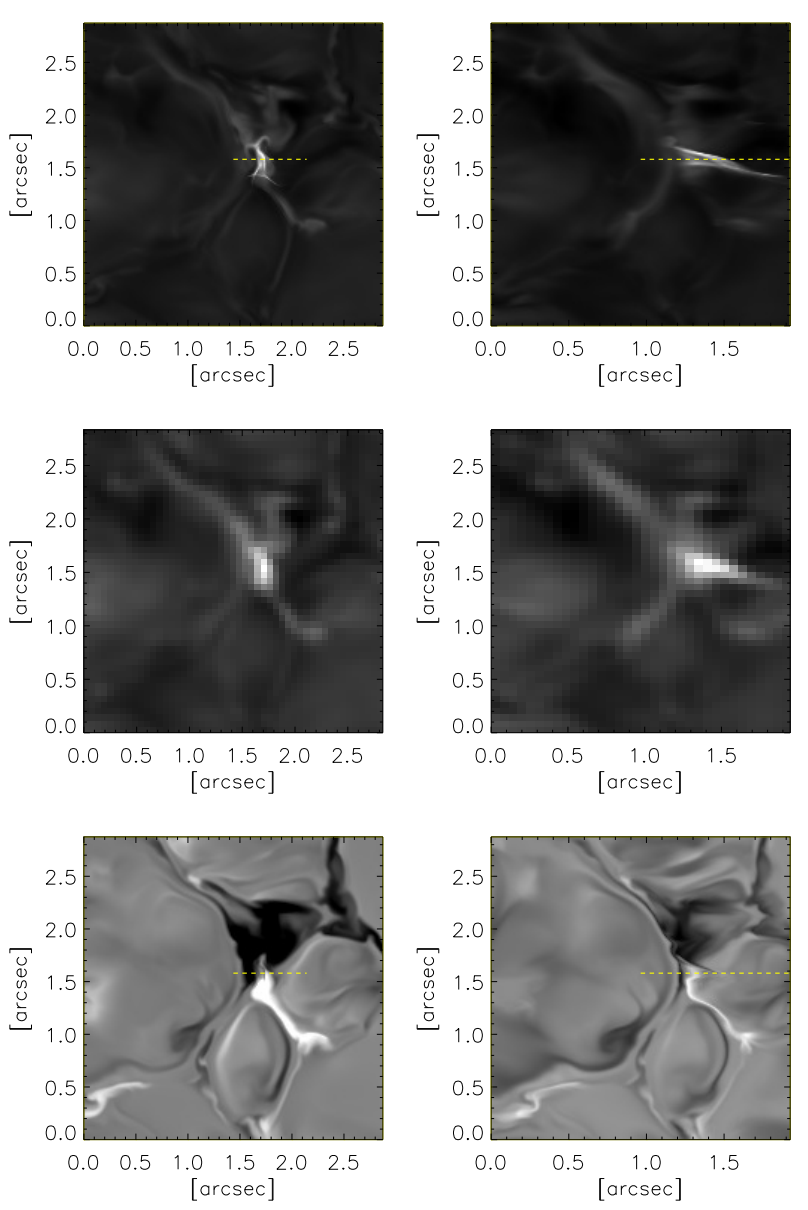

Fig. 3. Synthesized images observed at disk centre (first column) and at viewing angle $\theta=49^{\circ}$ and azimuthal angle $\phi=90^{\circ}$ (second column). Both columns are for time $t=62 \mathrm{~s}$ (third sample in Fig. 1, second row in Fig. 2). Top row: $\mathrm{H}_{\alpha}$ wing intensity at simulation resolution. Middle row: $\mathrm{H}_{\alpha}$ wing intensity at SST resolution. Bottom row: Fe I $6301 \AA$ magnetograms at simulation resolution. The yellow dashed lines in the top and bottom panels mark the locations of the vertical cuts shown in Figs. 4 and 5.

at the height where the EB-like feature reaches its highest temperature.

The simultaneous increase in temperature and density in the EB-like feature is present at the location where the apparent partial cancellation of the positive patch against the negative one takes place in Fig. 1. The corresponding field lines in Fig. 1 suggest closed loops between them, similar to the case presented in Cheung et al. (2010). The apparent cancellation and heating at this site suggests reconnection between the legs of a $\Omega$-loop as they approach or are being pushed towards each other.

The second row of Fig. 4 displays the same quantities but against mean radial optical depth $\tau_{5000}$ for the continuum at $\lambda=5000 \AA$ and adding contours that outline the contribution function to the radially emergent intensity in the $\mathrm{H}_{\alpha}$ wing, as defined in the centre panel of the bottom row. The first panel of the second row shows that the hot top of the EB-like feature sampled by the cut plane is optically thick in the $\mathrm{H}_{\alpha}$ wing, so that its lower parts are shielded from view. The lines of sight to the left of it sample a cool intergranular lane, while the lines of sight to the right sample the edge of a large hot granule. In both adjacent areas the $\mathrm{H}_{\alpha}$ wing originates much deeper, close to $\tau_{5000}=1$. This is expected since the cool upper photosphere of typical standard 

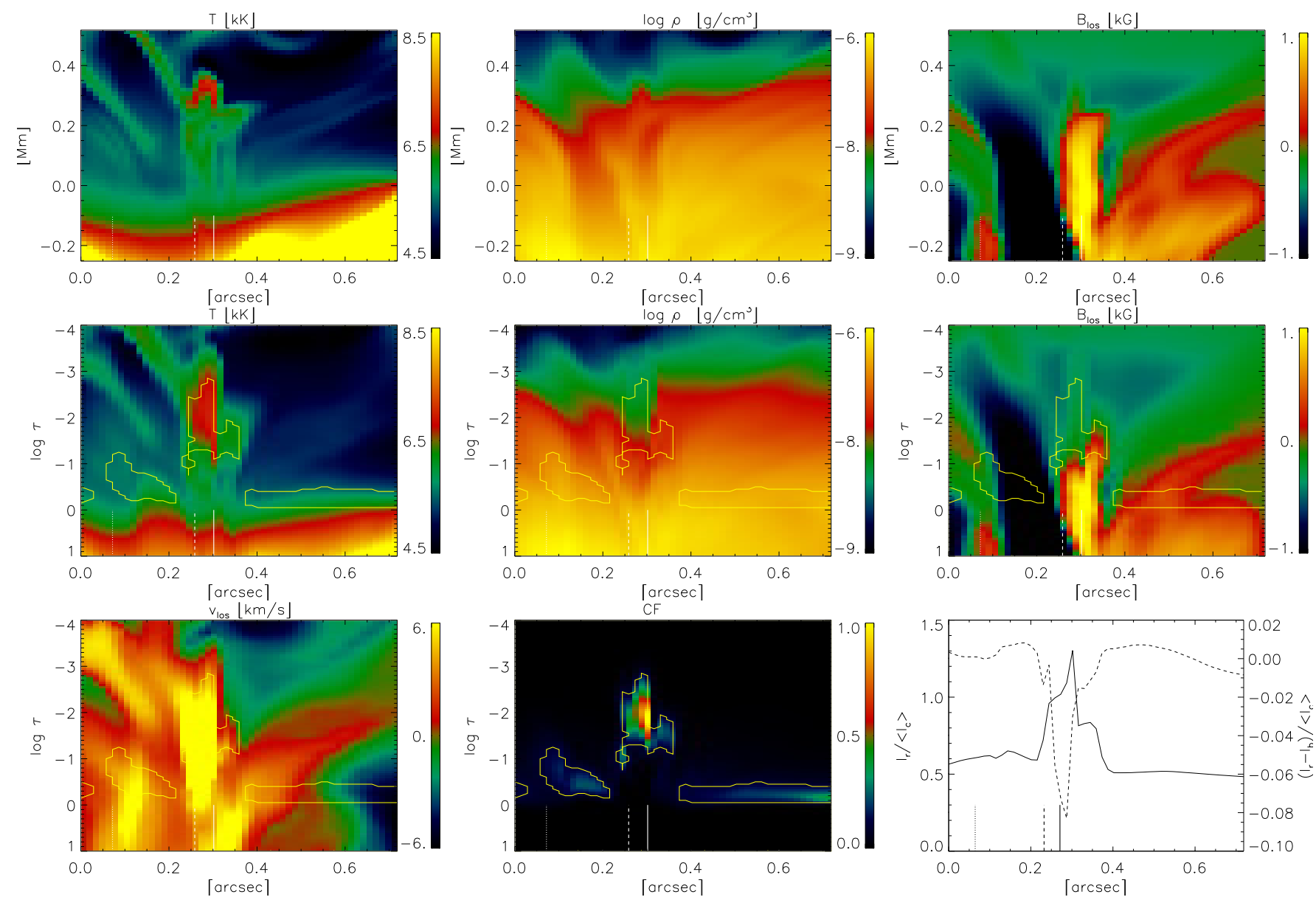

Fig. 4. Vertical cuts for viewing angle $\theta=0^{\circ}$ at the location shown in Fig. 3. First row: temperature, density, and line-of-sight component of the magnetic field against geometrical height. Second row: temperature, density, and line-of-sight field against optical depth. Third row: line-of-sight velocity, contribution function to $\mathrm{H}_{\alpha}$ line intensity at $\Delta \lambda=-0.11 \mathrm{~nm}$ from line centre, and $\mathrm{H}_{\alpha}$ wing intensity overplotted with Dopplergram signal (dashed). The yellow contours outline the formation height of the blue $\mathrm{H}_{\alpha}$ wing (central panel in the bottom row).
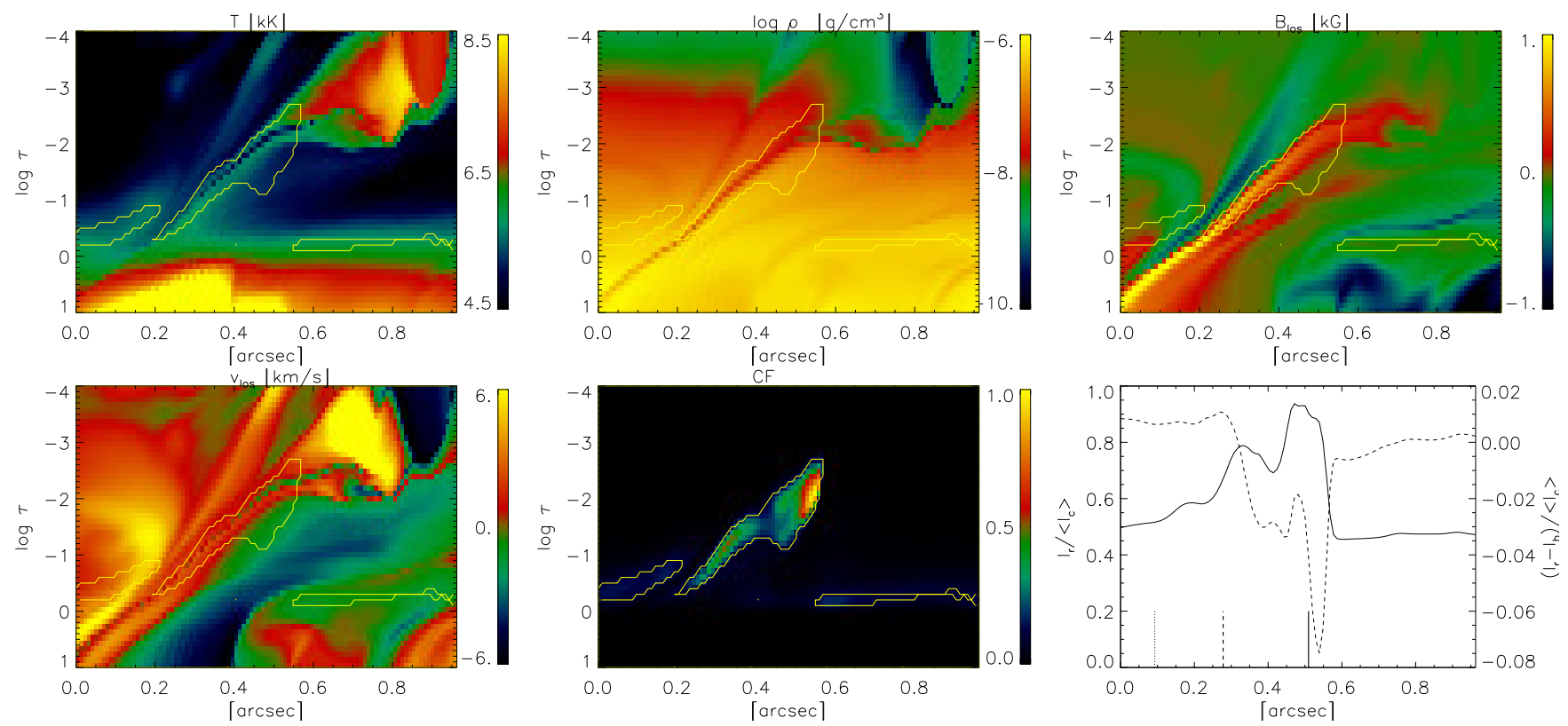

Fig. 5. Vertical plane through the skewed simulation with viewing angle $\theta=49^{\circ}$ and azimuthal angle $\phi=90^{\circ}$, along the cut segment specified in the right column of Fig. 3. The format is the same as in Fig. 4. The $\tau$ scales along the $y$-axes are now continuum optical depth at $5000 \AA$ along the slanted lines of sight through the cut plane (which has the solar limb to the right). The $x$-axis scales are the apparent distance over the solar surface including foreshortening. 
models contains no $\mathrm{H}_{\alpha}$ opacity, due to the low Boltzmann excitation of its lower level, whereas the higher temperature in the EB-like feature enhances the $\mathrm{H}_{\alpha}$ opacity substantially. We also note that the relatively large contrast that magnetic concentrations obtain in the blue $\mathrm{H}_{\alpha}$ wing thanks to its deep formation (Leenaarts et al. 2006) is indeed present in Fig. 3.

The density in the second panel of the second row shows a striking dip at the location of the density excess in the first row. This difference results from the transition from geometrical height to a $\tau_{5000}$ scale, and implies larger continuum opacity in the EB-like feature.

In the third panel of the second row, the $\mathrm{H} \alpha$-wing formation contour sits just above the abrupt end of sampling of the righthand magnetic concentration.

The first panel of the third row of Fig. 4 shows that the EB-like feature has a considerable downflow, and that an upflow lies above it and a bit to the side. The second panel defines the outline of the contribution to the emergent $\mathrm{H}_{\alpha}$-wing intensity used in the other panels. The third panel shows this emergent intensity (solid) across the cut, and also the subtractive Dopplergram signal whose deep dip corresponds well to the large downdraft seen at left.

This diagnosis indicates that in disk-centre viewing along the radial direction, only the top of an EB is seen, which shields what lies underneath. In addition, the third panel of Fig. 3 demonstrates that even at the SST resolution such a feature may not be easily distinguished from normal network concentrations.

Figure 5 is similar to Fig. 4 and shares its format, but diagnoses the skewed cube along the cut defined in the right column of Fig. 3. The cube is now rotated so that the direction of the line of sight is again vertical, as in Fig. 4. The direction towards the limb is to the right. The slanted view now also permits sampling of the lower parts of the EB-like feature. It results in extended elongated formation envelopes that reach down to the surface. This deeper-down sampling from the side indeed makes the bright corresponding feature in the right column of Fig. 3, which indeed displays flame morphology, as in the SST observations. The $\mathrm{H}_{\alpha}$-wing outlines the hot region and increases as the temperature increases, as shown in the bottom right panel of Fig. 5.

Finally, two positions along the features are chosen for quantitative comparison with 1D models. Figure 6 shows two examples that sample the EB-like feature at its middle and its top where the maximum temperature increase occurs. The height profiles show bumps where the temperature increases by 1500 to $3000 \mathrm{~K}$. This agrees with prediction from 1D modelling where the observed $\mathrm{H} \alpha$ wings are fit by introducing ad hoc perturbations of a static 1D standard model of the solar atmosphere. Furthermore, pixel 2 shows that in the slanted view representation, the top of the flame reaches $1 \mathrm{Mm}$ along the line of sight, similar to what some 1D EB models proposed. However, the rising temperature of $8000 \mathrm{~K}$ combined with the total hydrogen density of the order of $10^{14}-10^{15} \mathrm{~cm}^{-3}$ comes up short in comparison with the constraints given by Rutten (2016) that could insure the EB's visibility in a wide range of observables.

\subsection{Active region case}

To simulate an active region case, this time the flux sheet is introduced in a purely hydrodynamic set-up. The computational domain in this run is $12 \times 6 \times 3.5 \mathrm{Mm}$ at about $2 \mathrm{Mm}$ above the $\tau_{5000}=1$ surface and the same spatial resolution as in the quiet-Sun run. The width of the flux sheet and location where it is introduced are the same as in the quiet-Sun run, only in this
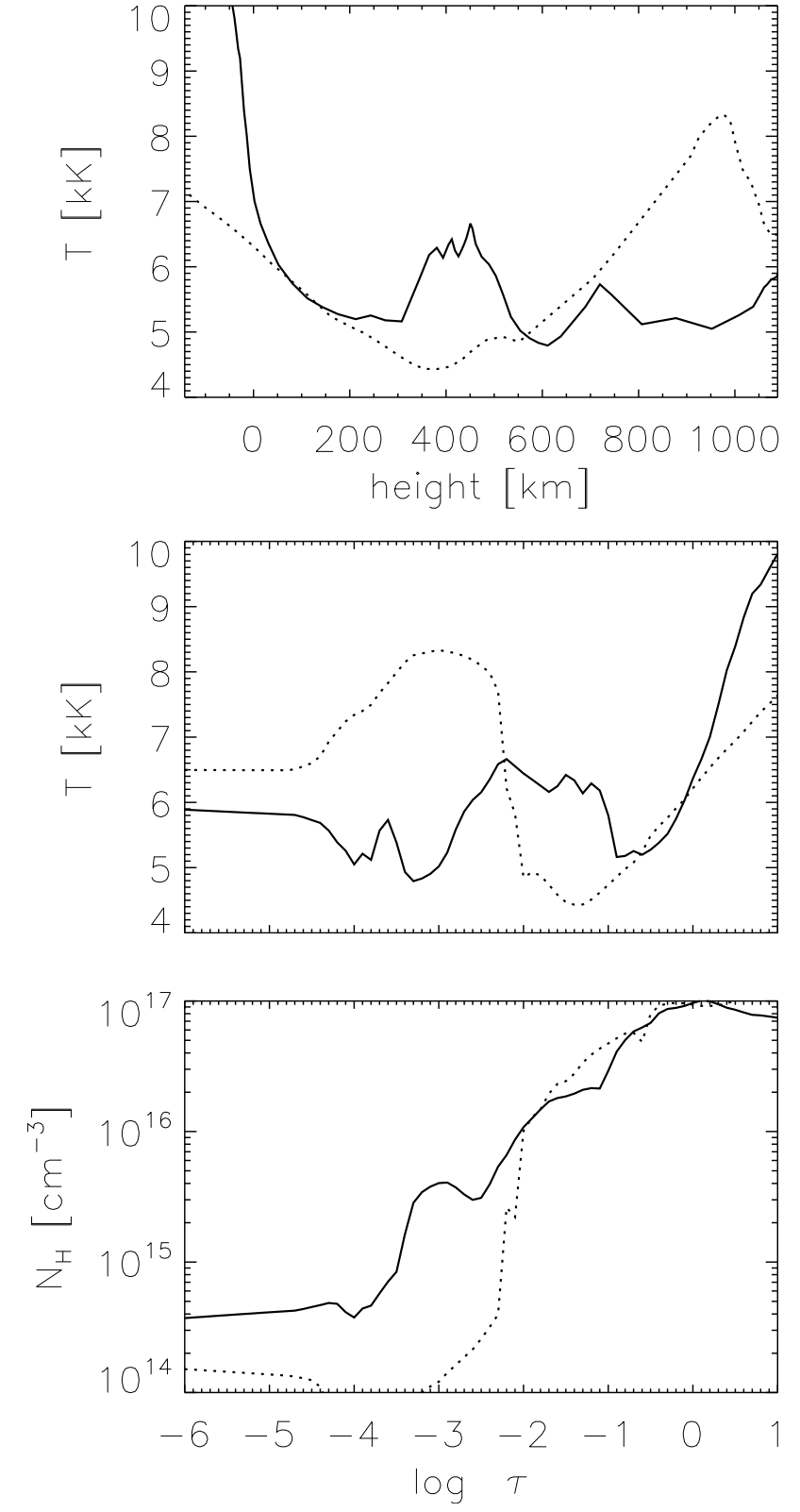

Fig. 6. Comparison of the atmospheric parameters at two locations: the middle (solid) and top (dashed) of the feature. Panels show temperature as a function of geometrical height (top panel) and optical depth (middle panel). The bottom panel shows total hydrogen density along the line of sight as a function of optical depth.

case the maximum field strength is $5000 \mathrm{G}$ instead of $200 \mathrm{G}$. This gives a maximum signed flux density of around $400 \mathrm{G}$, which roughly agrees with the values detected in the active regions. The flux sheet becomes undulated, due to convection, in such a way that crests and troughs are formed where convective uplows and downflows are present. Figure 7 shows a vertical field, $\mathrm{H} \alpha$-wing intensity, and the field topology at three moments during the emergence. At the first moment, the top of the flux sheet reached the convectively stable layer, while the $\mathrm{H} \alpha$-wing intensity map shows only weak brightenings at locations where reconnection takes place just at the surface. The first flame-like features appear a few minutes later when the emerged loops reach a few hundred $\mathrm{km}$ above the surface. The third instance shows the phase when the longest and brightest features appear. At this point the whole flux sheet has already emerged 

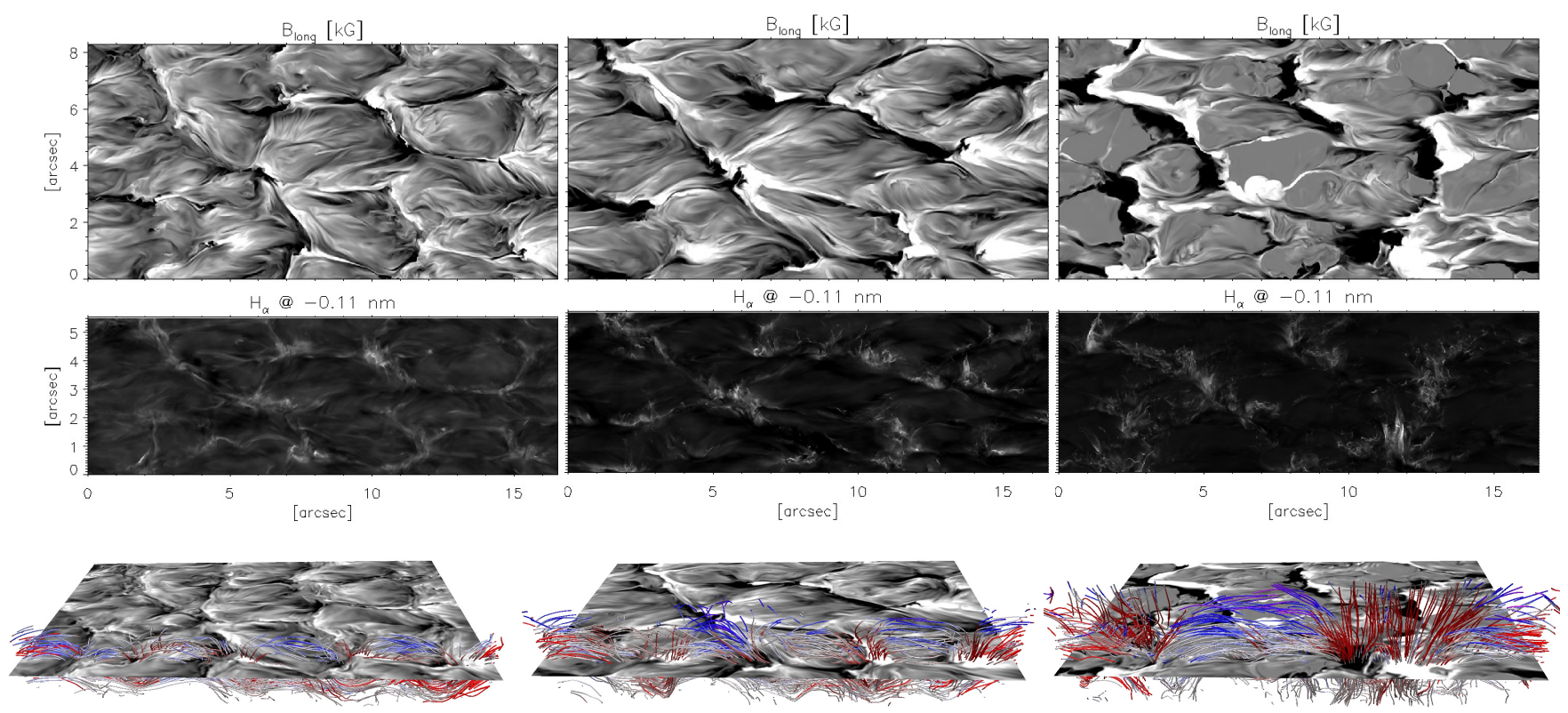

Fig. 7. Vertical component of magnetic field (top row), $\mathrm{H}_{\alpha}$ wing intensity (middle row), and field topology (bottom row) at $t=112,197$, and $549 \mathrm{~s}$ from left to right. The colour-coding of the field lines in the bottom panels corresponds to the vertical velocity with upflows being blue. Horizontal planes show the vertical component of the magnetic field at the surface. The magnetograms are cropped at $\pm 1 \mathrm{kG}$ and normalized $\mathrm{H}_{\alpha}$-wing intensity at 0.7 to 4.5 .
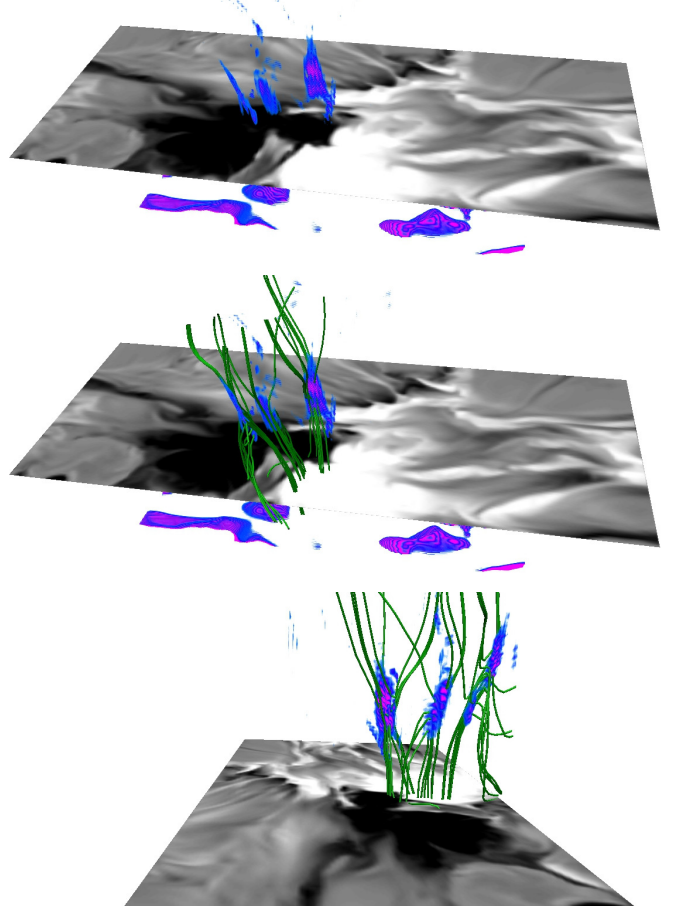

Fig. 8. Zoom in on a small part of the simulation domain at $t=549 \mathrm{~s}$ at $\left[11^{\prime \prime}, 1^{\prime \prime}\right]$. The horizontal panels show the vertical component of the magnetic field at the surface. The blue/purple rendering depicts the temperature in the range of 7500-9000 $\mathrm{K}$ and the green lines show the magnetic field configuration at these locations. The bottom panel shows the same region as the upper two, but from a different viewing angle.

and the non-perturbed granular pattern is restored. Similarly to the quiet Sun case, the most prominent EB-like features appear at the places where concentrated features of opposite polarities rush towards each other as the footpoints of the emerging loops travel horizontally.

In the active regions case, the morphology of EB-like features takes more complex forms than in the quiet Sun case, as a natural result of more intricate field topology. Figure 8 shows in detail how the "fork-shaped" feature visible at $\left[11^{\prime \prime}, 1\right.$ "] in the right-hand middle panel of Fig. 7 is generated. The temperature increase and the field configuration are similar to the quiet Sun case, only here it happens at three locations simultaneously. The largest jumps in temperatures occur near the temperature minimum where the temperature reaches $9000 \mathrm{~K}$. The field topology in all three locations is similar. It shows the bottom of the current sheets and reconnected $\Omega$-loops.

Finally, Fig. 9 shows how these features look in the continuum and the wings of $\mathrm{Na}$ I D1 and Mg I b2 lines. The chosen wavelength positions are the same as in observations obtained by Rutten et al. (2015). Although observations show that no signatures of EB can be visible in these lines, synthetic filtergrams show brightenings at the same locations as the $\mathrm{H} \alpha$ wings intensity image. Corresponding $\mathrm{H} \alpha$ wings in Fig. 7 show three EBlike features: at $\left[1^{\prime \prime}, 5^{\prime \prime}\right],\left[8^{\prime \prime}, 5^{\prime \prime}\right]$, and $\left[11^{\prime \prime}, 1^{\prime \prime}\right]$. The first two are also clearly visible in the $\mathrm{Na}$ I D1 and Mg I b2 lines. The third, the most pronounced one in the $\mathrm{H} \alpha$ wing can also be traced in the Na I D1 and Mg I b2 images, but only at its footpoint. The top and most of the fork-like shape is missed by the Na I D1 and $\mathrm{Mg} \mathrm{I}$ b2 lines. A comparison of the temperatures in these locations reveals that although the temperature increases at approximately the same heights, in the first two cases it stays below $8000 \mathrm{~K}$. In the third case, it is more than $1000 \mathrm{~K}$ higher, which comes into the temperature range where the $\mathrm{Mg}$ I and $\mathrm{Na} I$ populations drop rapidly (Rutten 2016).

\section{Conclusion}

Ellerman bomb-like features are simulated here by using the comprehensive 3D MHD code MURaM (Schüssler 2013). Two 

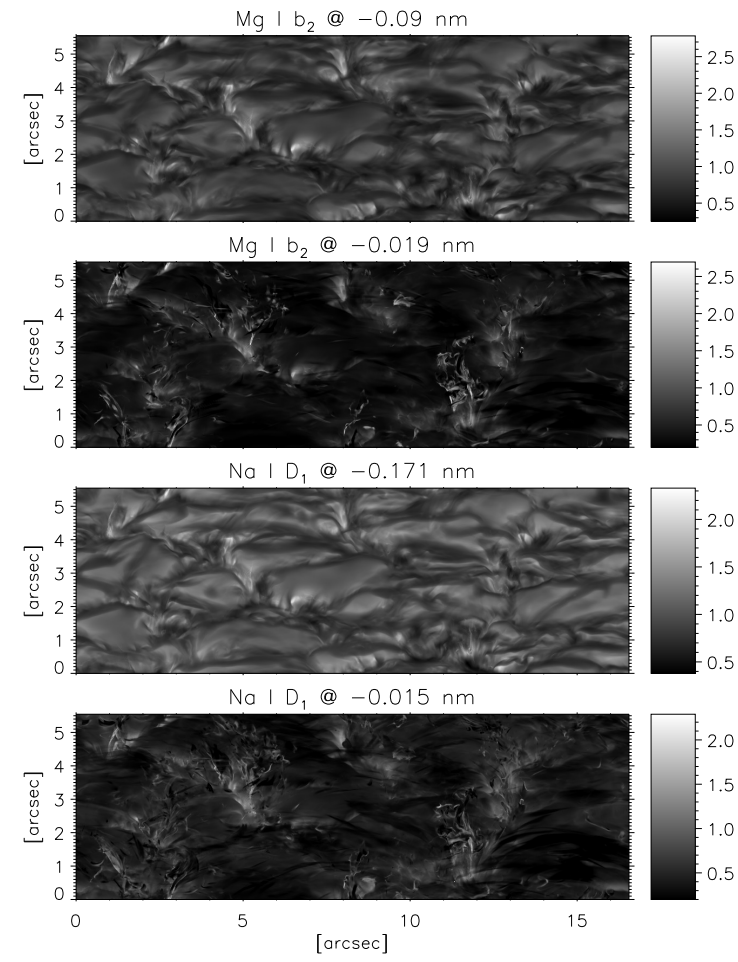

Fig. 9. Synthetic filtergrams in the continuum and the wing of Na I D1 and $\mathrm{Mg} \mathrm{I}$ b2 lines. The snapshot corresponds to the instant $t=549 \mathrm{~s}$ shown in Fig. 7.

different cases are presented: the quiet Sun case, which resembles the QSEBs conditions, and the active region case, which tries to reproduce the serpentine-like flux emergence. Although the simulation domain reaches $2 \mathrm{Mm}$ above the solar surface in the active region case, the analysis is limited to the lower atmospheric layers (temperature minimum and below) which are properly represented with this code. Going futher in the chromosphere requires taking into account more physics, such as radiative losses in the strongest chromospheric lines, nonequilibriums ionization of $\mathrm{H}$ and $\mathrm{He}$, etc.

The results presented here reveal that several characteristics of EB are replicated by the simulations:

- Firstly, EB-like brightenings coincide with hot and dense locations, in agreement with the predictions of 1D and 2D modellings. In the simulations, the hot clouds appear at or just below the temperature minimum which supports the claim that EBs are photospheric phenomena. The temperatures at these location are, in some cases, higher than $9000 \mathrm{~K}$, which is enough to reproduce the weak end of the EB spectrum; however, this does not exclude the possibility that higher temperatures could be produced higher up in the developed current sheets. The simulations show though that the wings of $\mathrm{H} \alpha$ always sample the low atmospheric layers in these runs. In the hottest cases, the features visible in the $\mathrm{H} \alpha$ wings will not be present in the $\mathrm{Na} \mathrm{I} \mathrm{D1} \mathrm{and} \mathrm{Mg} \mathrm{I} \mathrm{b2}$ filtergrams, which is in agreement with observations.

- Secondly, the simulated EB-like phenomena has the observed flame-like morphology with the base rooted in intergranular lanes. As shown in this study, the slanted view reveals the lower parts of the EB-like feature, which results in extended elongated formation envelopes that reach down to the surface. The simulated features assume the direction perpendicular to the limb. In some cases, however, depending on the viewing angle, the form can be more complex and the flame can be observed at an angle. These complex forms are more often found in the active region case.

- Thirdly, simulated EB features are caused by reconnection apparent as the cancellation of strong-field patches of opposite polarity that move together. At the layers sampled by $\mathrm{H} \alpha$-wings, the magnetic field topology always seem to be similar. The field lines there trace the base of the current sheet and the reconnected $\Omega$-loops. This is the same in both the quiet Sun and the active region cases. Although the opposite polarities are always present, this might not always be detected in observations, due to projection effects. The detection is further impaired with the decrease in spectral and spatial resolution and poor polarimetric sensitivity. This suggests that shearing magnetic field hypothesis (Watanabe et al. 2008; Vissers et al. 2013) might not be needed to explain the unipolar EB cases (Qiu et al. 2000; Georgoulis et al. 2002; Watanabe et al. 2008; Hashimoto et al. 2010).

- Finally, another similarity between the two runs is that the EB-like features do not appear in all regions where opposite polarities cancel out. They seem to appear in cases where opposite polarities approach each other at a reasonable high speed. As we showed in Danilovic et al. (2017), EBs form and last as long as the surface flows driving them together persist, and they can reappear or increase in brightness during re-emergence. This agrees with observations which also show that the EBs appear in the regions where the surface flows are the strongest (Vissers et al. 2013; Reid et al. 2016; Nelson et al. 2016), due to large-scale emergence or the moat flows. Based on this, it could also be argued that QSEBs are triggered during a rapid emergence on smaller scales.

Acknowledgements. This work has benefited from the discussions at the meeting "Solar UV bursts - a new insight to magnetic reconnection" at the International Space Science Institute (ISSI) in Bern. NASA's Astrophysics Data System (ADS) and the 3D visualization tool Vapor were extensively used in this study.

\section{References}

Archontis, V., \& Hood, A. W. 2009, A\&A, 508, 1469

Beeck, B., Collet, R., Steffen, M., et al. 2012, A\&A, 539, A121

Bello González, N., Danilovic, S., \& Kneer, F. 2013, A\&A, 557, A102

Berlicki, A., \& Heinzel, P. 2014, A\&A, 567, A110

Bruzek, A. 1968, Structure and Development of Solar Active Regions, 35, 293

Cheung, M. C. M., \& Cameron, R. H. 2012, ApJ, 750, 6

Cheung, M. C. M., Rempel, M., Title, A. M., \& Schüssler, M. 2010, ApJ, 720, 233

Danilovic, S., Cameron, R. H., \& Solanki, S. K. 2015, A\&A, 574, A28

Danilovic, S., Solanki, S. K., Barthol, P., et al. 2017, ApJS, 229, 5

Ellerman, F. 1917, ApJ, 46, 298

Fang, C., Tang, Y. H., Xu, Z., Ding, M. D., \& Chen, P. F. 2006, ApJ, 643, 1325

Frutiger, C. 2000, Ph.D. Thesis, Institute of Astronomy, ETH Zürich, Switzerland

Frutiger, C., Solanki, S. K., Fligge, M., \& Bruls, J. H. M. J. 2000, A\&A, 358, 1109

Georgoulis, M. K., Rust, D. M., Bernasconi, P. N., \& Schmieder, B. 2002, ApJ, 575,506

Grubecka, M., Schmieder, B., Berlicki, A., et al. 2016, A\&A, 593, A32

Hashimoto, Y., Kitai, R., Ichimoto, K., et al. 2010, PASJ, 62, 879

Hong, J., Ding, M. D., Li, Y., Fang, C., \& Cao, W. 2014, ApJ, 792, 13

Isobe, H., Tripathi, D., \& Archontis, V. 2007, ApJ, 657, L53

Kitai, R. 1983, Sol. Phys., 87, 135

Leenaarts, J., Rutten, R. J., Sütterlin, P., Carlsson, M., \& Uitenbroek, H. 2006, A\&A, 449, 1209

Libbrecht, T., Joshi, J., de la Cruz Rodríguez, J., Leenaarts, J., \& Asensio Ramos, A. 2017, A\&A, 598, A33 
Matsumoto, T., Kitai, R., Shibata, K., et al. 2008, PASJ, 60, 577

Nelson, C. J., Shelyag, S., Mathioudakis, M., et al. 2013, ApJ, 779, 125

Nelson, C. J., Doyle, J. G., \& Erdélyi, R. 2016, MNRAS, 463, 2190

Pariat, E., Aulanier, G., Schmieder, B., et al. 2004, ApJ, 614, 1099

Pariat, E., Aulanier, G., Schmieder, B., et al. 2006, Adv. Space Res., 38, 902

Pariat, E., Masson, S., \& Aulanier, G. 2009, ApJ, 701, 1911

Pariat, E., Masson, S., \& Aulanier, G. 2012, in 4th Hinode Science Meeting

Unsolved Problems and Recent Insights, ASP Conf. Ser., 455, 177

Peter, H., Tian, H., Curdt, W., et al. 2014, Science, 346, 1255726

Qiu, J., Ding, M. D., Wang, H., Denker, C., \& Goode, P. R. 2000, ApJ, 544, L157

Reid, A., Mathioudakis, M., Doyle, J. G., et al. 2016, ApJ, 823, 110

Rempel, M., Schüssler, M., \& Knölker, M. 2009, ApJ, 691, 640

Rezaei, R., \& Beck, C. 2015, A\&A, 582, A104

Rouppe van der Voort, L. H. M., Rutten, R. J., \& Vissers, G. J. M. 2016, A\&A, 592, A100

Rutten, R. J. 2016, A\&A, 590, A124

Rutten, R. J., Vissers, G. J. M., Rouppe van der Voort, L. H. M., Sütterlin, P., \& Vitas, N. 2013, J. Phys. Conf. Ser., 440, 012007
Rutten, R. J., Rouppe van der Voort, L. H. M., \& Vissers, G. J. M. 2015, ApJ, 808,133

Schmieder, B., Archontis, V., \& Pariat, E. 2014, Space Sci. Rev., 186, 227

Schüssler, M. 2013, Solar and Astrophysical Dynamos and Magnetic Activity, IAU Symp., 294, 95

Socas-Navarro, H., Martínez Pillet, V., Elmore, D., et al. 2006, Sol. Phys., 235, 75

Tian, H., Xu, Z., He, J., \& Madsen, C. 2016, ApJ, 824, 96

Vissers, G. J. M., Rouppe van der Voort, L. H. M., \& Rutten, R. J. 2013, ApJ, 774,32

Vissers, G. J. M., Rouppe van der Voort, L. H. M., Rutten, R. J., Carlsson, M., \& De Pontieu, B. 2015, ApJ, 812, 11

Uitenbroek, H. 2001, ApJ, 557, 389

Vögler, A., Bruls, J. H. M. J., \& Schüssler, M. 2004, A\&A, 421, 741

Vögler, A., Shelyag, S., Schüssler, M., et al. 2005, A\&A, 429, 335

Watanabe, H., Kitai, R., Okamoto, K., et al. 2008, ApJ, 684, 736

Watanabe, H., Vissers, G., Kitai, R., Rouppe van der Voort, L., \& Rutten, R. J. 2011, ApJ, 736, 71 